\title{
Crescimento inicial do cafeeiro Rubi em resposta a doses de nitrogênio, fósforo e potássio e a regimes hídricos ${ }^{(1)}$
}

\author{
Rodrigo Barbosa Nazareno(2), Carlos Alberto da Silva Oliveira ${ }^{(2)}$, Claudio Sanzonowicz ${ }^{(3)}$, \\ João Batista Ramos Sampaio(3), Júlio César Pereira da Silva ${ }^{(2)}$ e Antônio Fernando Guerra ${ }^{(3)}$
}

Resumo - O objetivo deste trabalho foi avaliar o crescimento da parte aérea do cafeeiro (Coffea arabica L.) cultivar Rubi MG 1192 submetido a três doses de N, P e K e dois regimes hídricos durante o primeiro ano após o transplante, em 20 de novembro de 2000. O crescimento da planta foi avaliado aos 134, 196, 236, 284, 334 e 383 dias após o transplante (DAT). Houve resposta ao $\mathrm{N}$ e ao K no crescimento em número de ramos plagiotrópicos por planta, ao passo que no número de nós com gemas por planta, observou-se resposta apenas ao nitrogênio. Não houve resposta ao N, P e K no aumento da massa seca da parte aérea e no índice de área foliar. Além de mostrar efeito significativo no crescimento do cafeeiro, a irrigação antecipou o rápido crescimento para julho (236 DAT) proporcionando plantas mais vigorosas. Nas plantas não-irrigadas, o rápido crescimento ocorreu em meados de outubro (334 DAT). Entretanto, a irrigação não impediu a queda na taxa de crescimento durante o inverno. O desenvolvimento das gemas em frutos ou ramos secundários nas plantas não-irrigadas alterou a distribuição de matéria seca e reduziu o crescimento do caule, ramos e folhas.

Termos para indexação: Coffea arabica, irrigação, adubação, déficit hídrico, solo de cerrado.

Initial growth of Rubi coffee plant in response to nitrogen, phosphorus and potassium and water regimes

\begin{abstract}
The objective of this work was to evaluate shoot growth of coffee trees (Coffea arabica L.) cultivar Rubi MG 1192 under three N, P and K doses and two water regimes during the first year after transplanting, starting on the $20^{\text {th }}$ November 2000 . The growth was evaluated at $134,196,236$, 284, 334 and 383 days after transplanting (DAT). Nitrogen and K affected the number of plagiotropic branches per plant. Nevertheless, only $\mathrm{N}$ affected the number of nodes with axillary buds. There was no growth response to $\mathrm{N}, \mathrm{P}$, and $\mathrm{K}$ in terms of the total shoot dry mass and leaf area index during the experimental period. Irrigated treatments presented the best plant growth results, provided more vigorous plants and anticipated the fast growth phase to July (236 DAT), but could not avoid growth rate decrease during the winter. The fast plant growth phase of non-irrigated plants occurred by the middle of October (334 DAT); in these treatments, the development of buds into fruits or secondary branches altered the dry matter distribution and reduced stem, branches and leaves growth.
\end{abstract}

Index terms: Coffea arabica, irrigation, fertilizer application, water deficit, cerrado soils.

\section{Introdução}

A região dos Cerrados apresenta grande potencial para a produção de café com objetivos comerciais

\footnotetext{
(1) Aceito para publicação em 26 de maio de 2003 .

(2) Universidade de Brasília, Fac. de Agronomia e Medicina Veterinária, Caixa Postal 04508, CEP 70919-970 Brasília, DF. E-mail: rodrigonazareno@yahoo.com.br, dasilvao@unb.br, jcpereira@br.inter.net

(3) Embrapa-Centro de Pesquisa Agropecuária dos Cerrados, Caixa Postal 08223, CEP 73301-970 Planaltina, DF. E-mail: sanzo@cpac.embrapa.br, sampaio@cpac.embrapa.br, guerra@cpac.embrapa.br
}

e empresariais, e o uso de tecnologias tais como cultivares adaptadas, fertilização adequada do solo, controle fitossanitário e mecanização pode garantir elevada produtividade e qualidade do produto. No entanto, fatores adversos à cafeicultura nos Cerrados, como a má distribuição de chuvas no decorrer do ano e a deficiência hídrica no período da seca, parecem evidenciar a necessidade de irrigação, visando reduzir riscos.

Há diversos nutrientes necessários ao bom desenvolvimento do cafeeiro, no entanto, a falta de $\mathrm{N}$ é que mais limita seu crescimento e produção (Vaast 
et al., 1998). As exigências de K são equivalentes às de N (Silva et al., 1999). O P, apesar de ser essencial para a produção dos frutos e para o rápido crescimento do cafeeiro novo, é um nutriente pouco exigido em comparação com o N e K (Guimarães, 1986).

Tesha \& Kumar (1979), trabalhando com cafeeiros da cultivar K7 com um ano de idade, em vasos, constataram que a absorção de N, P e K estava relacionada com as quantidades dos fertilizantes aplicadas e com o teor de água no solo. A aplicação de $\mathrm{Ne}$ $\mathrm{K}$ em solo com $50 \%$ da capacidade de campo aumentou o crescimento da planta. Na presença de déficit hídrico, o N e o K não estimularam o crescimento. Após o reinício das irrigações, houve crescimento das plantas que foram originalmente mantidas em uma baixa umidade de solo.

Winston et al. (1992) estudaram durante cinco anos o efeito de N e K no crescimento e na produção do café no Norte do Quênia, num experimento irrigado composto das cultivares K2 e Kairi Local Typica, de porte alto, e das cultivares CR33, CR35 e B61, de porte baixo. Não foram encontradas diferenças significativas nos parâmetros de crescimento vegetal e de produção de grãos com o aumento das doses de $\mathrm{N}$ e potássio. Entretanto, existem poucos trabalhos sobre crescimento inicial do cafeeiro influenciado pela nutrição de N, P ou K e pelo teor de água no solo em cultivos adensados, principalmente em solos de Cerrados.

O crescimento do cafeeiro no Sudeste brasileiro é reduzido no período frio e seco e, no período quente e chuvoso, a planta apresenta um rápido crescimento. Tal sazonalidade do crescimento pode ser influenciada pela amplitude de variação da temperatura do ar (Mota, 1988; Amaral, 1991; Barros et al., 1997; Matta et al., 1999; Silva, 2000), pela redução da condutância estomática durante o período frio e seco (Mota, 1988; Barros et al., 1997; Silva, 2000) e pela redução do fotoperíodo (Amaral, 1991). No entanto, Barros et al. (1997) não constataram crescimento adicional quando mantido o fotoperíodo de 14 horas. Em estudo semelhante, Mota (1988) observou que mesmo sob temperaturas baixas, os dias mais longos promoveram reduções na queda das folhas.

Por sua vez, a quantidade de água no solo durante a fase de crescimento intenso influencia o crescimento vegetal. Assim, Gervásio \& Lima (1998) cons- tataram que na fase inicial de formação do cafeeiro, cultivar Icatu MG 3282, com seis meses de idade, plantado em vasos, o aumento da umidade do solo acelerou o desenvolvimento da planta.

Ao realizarem fertilização suplementar com $\mathrm{N}$ via água de irrigação, durante a estação fria e seca, Amaral (1991) e Matta et al. (1999) observaram que este tratamento não impediu a redução do crescimento da cultivar Catuaí Vermelho durante os meses de inverno e tampouco alterou o padrão sazonal de crescimento. Porém o N suplementar propiciou maior crescimento de área foliar e ramos plagiotrópicos no período quente e úmido.

Os frutos, por demandarem prioritariamente os fotoassimilados, podem influenciar o crescimento do cafeeiro (Rena \& Maestri, 2000). Cafeeiros adultos que tiveram os frutos removidos não alteraram o padrão de crescimento, embora apresentassem maiores taxas de crescimento (Amaral 1991).

O objetivo deste trabalho foi avaliar o crescimento da parte aérea do cafeeiro cultivar Rubi MG 1192, em plantio adensado, submetido a três doses de N, P e $\mathrm{K}$ e dois regimes hídricos durante o primeiro ano após o transplante.

\section{Material e Métodos}

Entre as cultivares adaptadas às condições dos Cerrados, optou-se por utilizar a cultivar Rubi MG 1192, de alto vigor vegetativo, maturação precoce e uniforme, e que não apresenta seca de ponteiros (Mendes, 1999). Por possuir porte baixo e arquitetura adequada, é indicada para plantios circulares e em sistema de renque adensado.

O trabalho foi realizado no campo experimental da Embrapa-Centro de Pesquisa Agropecuária dos Cerrados, situado à latitude $15^{\circ} 34^{\prime} 30^{\prime \prime} \mathrm{S}$ e longitude $47^{\circ} 42^{\prime} 30^{\prime \prime} \mathrm{W}$, com altitude média de $1.000 \mathrm{~m}$. Segundo a classificação de Köppen, o clima é do tipo Aw, tropical chuvoso de inverno seco, apresentando deficiência hídrica em torno de $168 \mathrm{~mm}$, necessitando de irrigação suplementar para a cultura do cafeeiro. O solo é classificado como Latossolo Vermelho, fase cerrado com textura argilosa.

Segundo metodologia adotada pela Embrapa (1997), as características químicas do solo, na camada de $0-20 \mathrm{~cm}$, apresentaram valores do $\mathrm{pH}$ em água, 5,2; $\mathrm{pH}$ em $\mathrm{CaCl}_{2}$, 4,63; $\mathrm{Al}^{3+}, 4,3 \mathrm{mmol}_{\mathrm{C}} / \mathrm{dm}^{3} ; \mathrm{Ca}^{2+}, 22,9 \mathrm{mmol}_{\mathrm{c}} / \mathrm{dm}^{3} ; \mathrm{Mg}^{2+}$, $8,3 \mathrm{mmol}_{\mathrm{c}} / \mathrm{dm}^{3} ; \mathrm{H}+\mathrm{Al}, 76,0 \mathrm{mmol}_{\mathrm{c}} / \mathrm{dm}^{3} ; \mathrm{P}, 1,4 \mathrm{mg} / \mathrm{dm}^{3} ; \mathrm{K}$, $61,2 \mathrm{mg} / \mathrm{dm}^{3} ; \mathrm{Cu}, 0,85 \mathrm{mg} / \mathrm{dm}^{3} ; \mathrm{Fe}, 33,4 \mathrm{mg} / \mathrm{dm}^{3} ; \mathrm{Mn}$, $2,61 \mathrm{mg} / \mathrm{dm}^{3} ; \mathrm{Zn}, 1,01 \mathrm{mg} / \mathrm{dm}^{3}$ e saturação por $\mathrm{Al}, 12 \%$. 
A análise física $(0-20 \mathrm{~cm})$ apresentou valores de $600 \mathrm{~g} / \mathrm{kg}$ de argila; $116 \mathrm{~g} / \mathrm{kg}$ de silte; $47 \mathrm{~g} / \mathrm{kg}$ de areia grossa e $236 \mathrm{~g} / \mathrm{kg}$ de areia fina. Na camada de $20-40 \mathrm{~cm}$, os valores foram de $635 \mathrm{~g} / \mathrm{kg}$ de argila; $94 \mathrm{~g} / \mathrm{kg}$ de silte; $43 \mathrm{~g} / \mathrm{kg}$ de areia grossa e $23 \mathrm{~g} / \mathrm{kg}$ de areia fina. Com base nas análises físico-químicas da área, aplicaram-se 4,1 t/ha de calcário dolomítico, a fim de elevar a saturação por bases a $60 \%$; 4,0 t/ha de gesso agrícola e $280 \mathrm{~kg} / \mathrm{ha}$ de $\mathrm{P}_{2} \mathrm{O}_{5}$ (superfosfato triplo).

O delineamento experimental nos regimes hídricos foi o de blocos ao acaso para os fatores adubação e irrigação, tendo a época de avaliação como subparcela, e quatro repetições.

O transplante das mudas de café foi realizado no dia 20 de novembro de 2000 no espaçamento 2,8x0,5 m, caracterizando plantio adensado. Cada parcela foi constituída de três fileiras com oito plantas em uma área de $33,6 \mathrm{~m}^{2}$; 36 parcelas foram alocadas sob irrigação e outras 36 sob regime hídrico sem irrigação. O ajuste de espaçamento foi determinado segundo Androcioli Filho (1994), que considera o diâmetro da copa do cafeeiro adulto e o espaço livre desejado entre as fileiras.

Os nove tratamentos de N, Pe K em cada regime hídrico (irrigado e sem irrigação) foram constituídos da combinação de três doses de $\mathrm{N}(12,5,62,5$ e $200 \mathrm{~kg} / \mathrm{ha})$ com doses fixas de $\mathrm{P}_{2} \mathrm{O}_{5}$ e $\mathrm{K}_{2} \mathrm{O}$ (214,3 e 107,1 kg/ha, respectivamente); da combinação de três doses de $\mathrm{P}_{2} \mathrm{O}_{5}(100,200$ e $500 \mathrm{~kg} / \mathrm{ha})$ com doses fixas de $\mathrm{Ne} \mathrm{K}_{2} \mathrm{O}$ (114,3 e 107,1 kg/ha, respectivamente) e da combinação de três doses de $\mathrm{K}_{2} \mathrm{O}(12,5$, $62,5$ e $200 \mathrm{~kg} / \mathrm{ha})$ com doses fixas de $\mathrm{N}_{\text {e }} \mathrm{P}_{2} \mathrm{O}_{5}(114,3$ e $214,3 \mathrm{~kg} / \mathrm{ha}$, respectivamente). As fontes utilizadas foram: uréia $(\mathrm{N})$, superfosfato triplo $(\mathrm{P})$, cloreto de potássio $(\mathrm{K})$, bórax (3,6 kg/ha de B), sulfato de cobre (3,6 kg/ha de $\mathrm{Cu}$ ), sulfato de zinco $(7,1 \mathrm{~kg} / \mathrm{ha}$ de $\mathrm{Zn})$ e sulfato de manganês (7,1 kg/ha de Mn). O manejo da adubação foi comum aos dois regimes hídricos, e os nutrientes, à exceção das doses variáveis de N, P e K, fornecidos conforme recomendação proposta por Raij et al. (1996). A adubação com P e micronutrientes foi realizada por ocasião do plantio, aplicando os fertilizantes no fundo do sulco. A aplicação de N e $\mathrm{K}$ foi realizada em cobertura dividida em quatro parcelamentos a cada 30 dias durante o período chuvoso (20 de dezembro de 2000, 20 de janeiro de 2001, 20 de fevereiro de 2001 e 20 de março de 2001), em faixa, na projeção da copa das plantas.

O manejo de irrigação por pivô central foi feito usando medidas do conteúdo de água no solo pela leitura da sonda Theta Probe tipo ML2X da Delta-T Devises instalada a $0,1 \mathrm{~m}$ de profundidade, local de maior densidade de raízes. Sempre que a leitura acusasse valores em torno de $0,29 \mathrm{~cm}^{3} / \mathrm{cm}^{3}$ (fator de disponibilidade de 50\%), valor representativo da tensão de água no solo de $50 \mathrm{kPa}$, procedia-se à aplicação de água para que o perfil de $40 \mathrm{~cm}$ do solo atingisse a condição de capacidade de campo $\left(0,35 \mathrm{~cm}^{3} / \mathrm{cm}^{3}\right)$. Utilizaram-se os dados obtidos na estação climatológica local para relacionar as necessidades hídricas das plantas e seu crescimento com as condições atmosféricas (Figura 1).

As respostas aos tratamentos de N, P e K e regimes hídricos foram avaliadas após o término das adubações de cobertura e uma planta por tratamento foi coletada e analisada nas seguintes épocas: 3 de abril, 134 DAT; 4 de junho, 196 DAT; 14 de julho, 236 DAT; 31 de agosto, 284 DAT; 20 de outubro, 334 DAT; e 9 de dezembro, 383 DAT. Os seguintes parâmetros foram analisados: número de ramos plagiotrópicos e de nós com gemas axilares (de 31 de agosto em diante, as gemas tornaram-se objeto de estudo e, no decorrer do tempo, se desenvolveram em ramificações secundárias e em frutos, especialmente nas plantas sem irrigação), índice de área foliar e massa da parte aérea seca, que foi o somatório das massas do caule, ramos, gemas e folhas. Foram determinadas as taxas médias de crescimento do índice de área foliar e da massa da parte aérea seca e a distribuição de matéria seca na parte aérea da planta.

A coleta dos cafeeiros foi processada ao acaso cortandoos imediatamente acima da inserção radicular. O número de ramos plagiotrópicos e de nós com gemas foram determinados por contagem direta. A área foliar foi determinada utilizando-se o aparelho Automatic Area Meter da Hayashi Denkoh Co Ltda. Posteriormente, as várias partes do cafeeiro foram acondicionadas em sacos de papel separadamente $\mathrm{e}$ secadas em estufa, com ventilação forçada a $60^{\circ} \mathrm{C}$, até que atingissem peso constante, determinando-se a massa.

Os dados obtidos foram submetidos à análise de variância e as médias comparadas pelo teste de Duncan a $5 \%$ de probabilidade. As variáveis que apresentaram coeficiente de variação elevado tiveram os dados observados transformados pela expressão $\mathrm{Y}=(\mathrm{X}+1)^{0,5}$. Curvas de crescimento das plantas em ambos regimes hídricos foram estabelecidas escolhendo-se os melhores coeficientes de determinação.

\section{Resultados e Discussão}

Houve resposta significativa para a interação entre adubação e regime hídrico em relação ao número de ramos plagiotrópicos por planta (Tabela 1). Em ambos regimes hídricos, os tratamentos que receberam $\mathrm{K}_{2} \mathrm{O}$ e, principalmente, $\mathrm{N}$ proporcionaram maiores amplitudes nas respostas que os tratamen- 
tos com $\mathrm{P}_{2} \mathrm{O}_{5}$. Houve resposta até a dose de $200 \mathrm{~kg} / \mathrm{ha}$ de $\mathrm{N}$ e até $62,5 \mathrm{~kg} / \mathrm{ha}$ de $\mathrm{K}_{2} \mathrm{O}$ sob irrigação. Não se constatou resposta às doses de $\mathrm{K}_{2} \mathrm{O}$ associadas a regime hídrico não-irrigado. A ausência de respostas nos tratamentos com $\mathrm{P}_{2} \mathrm{O}_{5}$ pode ser explicada em razão da dose de $\mathrm{P}$ prévia ao plantio $\left(40 \mathrm{~g}\right.$ de $\mathrm{P}_{2} \mathrm{O}_{5} /$ planta) e do $\mathrm{P}$ da menor dose (14 g de $\mathrm{P}_{2} \mathrm{O}_{5} /$ planta). Embora elas estejam abaixo das recomendadas por Guimarães et al. (1999), são adequadas para o crescimento e desenvolvimento da planta no primeiro ano. Pode-se, assim, afirmar que o cafeeiro apresenta maior resposta à doses crescentes de $\mathrm{N}$ e $\mathrm{K}_{2} \mathrm{O}$, elementos bastante necessários para o crescimento desta planta (Vaast et al., 1998; Silva et al., 1999) no primeiro ano.

Em relação ao crescimento em número médio de nós com gemas por planta, foi constatada diferença significativa em virtude do regime hídrico, com 76 nós em plantas irrigadas contra 46 nós em plantas sem irrigação. Independentemente do regime hídrico, as plantas que receberam $200 \mathrm{~kg} / \mathrm{ha}$ de $\mathrm{N}$ e 200 e $500 \mathrm{~kg} / \mathrm{ha}$ de $\mathrm{P}_{2} \mathrm{O}_{5}$ apresentaram maiores médias. As menores médias foram observadas nos tratamentos com $12,5 \mathrm{~kg} / \mathrm{ha}$ de $\mathrm{K}_{2} \mathrm{O}$ e $12,5 \mathrm{~kg} / \mathrm{ha}$ de $\mathrm{N}$ (Tabela 1), evidenciando que estes elementos são fundamentais no aparecimento das rosetas.

$\mathrm{O}$ índice de área foliar e a massa da parte aérea seca não responderam significativamente ao $\mathrm{N}$, ao $\mathrm{P}$ $\mathrm{e}$ ao $\mathrm{K}$ aplicados nos cafeeiros em ambos regimes hídricos durante o período de avaliação, sugerindo que as doses mínimas disponíveis foram adequadas para a planta. Ausência de respostas às doses crescentes de N, P e K no crescimento do cafeeiro foram encontradas por Winston et al. (1992). Por outro lado, o crescimento de mudas de cafeeiro em altura, em área foliar e em número de nós aumentou pela aplicação de $\mathrm{N}$ e $\mathrm{K}$ associada a teores adequados de água no solo (Tesha \& Kumar, 1979).

Houve interação entre regime hídrico e época de avaliação. Independentemente do regime hídrico, ocorreu aumento significativo do índice de área foliar e da massa seca da parte aérea a partir de 4 de junho (Tabela 2). Isto evidenciou claramente fases de crescimento lento e ativo ou acelerado, provavelmente, por causa da diferença de temperatura do ar. O índice de área foliar das plantas irrigadas diferiu significativamente das não irrigadas a partir de 14 de julho. Em relação à massa da parte aérea seca da planta, diferenças significativas só foram observadas a partir de 31 de agosto. No período de inverno, em que as precipitações foram baixas (Figura 1), a água facilmente disponível para as plantas irrigadas favoreceu a antecipação deste crescimento.

A irrigação não impediu a redução do crescimento no período de junho a julho (Figura 2), que coincidiu com o período de maiores amplitudes nas médias mensais de temperatura do ar e com as menores médias mensais de umidade relativa (Figura 1). No período de julho a agosto, com a elevação da temperatura mínima e da umidade relativa do ar, foram observadas maiores taxas de crescimento nas plantas irrigadas e, menores taxas nas sem irrigação. Conforme Rena \& Maestri (1986), a periodicidade de crescimento do cafeeiro em várias regiões do mun-

Tabela 1. Número de ramos plagiotrópicos e de nós com gemas por planta do cafeeiro Rubi MG 1192 em razão de doses crescentes de $\mathrm{N}, \mathrm{P}_{2} \mathrm{O}_{5}$ e $\mathrm{K}_{2} \mathrm{O}$ (kg/ha), em regimes hídricos irrigado e não-irrigado aos 383 dias após o transplante ${ }^{(1)}$.

\begin{tabular}{|c|c|c|c|c|c|c|c|c|c|}
\hline \multirow[t]{2}{*}{ Tratamento } & \multicolumn{3}{|c|}{$\mathrm{N}^{(2)}$} & \multicolumn{3}{|c|}{$\mathrm{P}_{2} \mathrm{O}_{5}{ }^{(3)}$} & \multicolumn{3}{|c|}{$\mathrm{K}_{2} \mathrm{O}^{(4)}$} \\
\hline & 12,5 & 62,5 & 200 & 100 & 200 & 500 & 12,5 & 62,5 & 200 \\
\hline & \multicolumn{9}{|c|}{ Ramos plagiotrópicos } \\
\hline Irrigado & $14,7 \mathrm{cde}$ & $15,2 \mathrm{cde}$ & $18,0 \mathrm{a}$ & $16,4 \mathrm{abcd}$ & $16,5 \mathrm{abcd}$ & $16,7 \mathrm{abc}$ & $14,8 \mathrm{cde}$ & $15,7 \mathrm{bcd}$ & $17,3 \mathrm{ab}$ \\
\hline Não-irrigado & $13,5 \mathrm{e}$ & $16,1 \mathrm{abcd}$ & $15,1 \mathrm{cde}$ & $15,2 \mathrm{cde}$ & $15,5 \mathrm{bcde}$ & $14,5 \mathrm{de}$ & $15,0 \mathrm{cde}$ & $14,9 \mathrm{cde}$ & $14,8 \mathrm{cde}$ \\
\hline Médias & \multicolumn{9}{|c|}{ Nós com gemas } \\
\hline
\end{tabular}

(1)Para uma mesma variável, médias seguidas da mesma letra não diferem entre si pelo teste de Duncan, a $5 \%$ de probabilidade. (2) $\mathrm{As}$ doses de $\mathrm{P}_{2} \mathrm{O}_{5}$ e $\mathrm{K}_{2} \mathrm{O}$ foram de 214,3 e 107,3 kg/ha, respectivamente. ${ }^{(3)}$ As doses de $\mathrm{N}_{\mathrm{K}} \mathrm{K} 2 \mathrm{O}$ foram de 114,3 e 107,3 kg/ha, respectivamente. ${ }^{(4)} \mathrm{As}$ doses de $\mathrm{N}$ e $\mathrm{P}_{2} \mathrm{O}_{5}$ foram de 114,3 e $214,3 \mathrm{~kg} / \mathrm{ha}$, respectivamente. 
Tabela 2. Médias do índice de área foliar (IAF) e da massa da parte aérea seca (MS) por planta do cafeeiro Rubi MG 1192 em razão dos dias após o transplante (DAT) e dos regimes hídricos e equações ajustadas para o período de estudo ${ }^{(1)}$.

\begin{tabular}{|c|c|c|c|c|c|c|c|}
\hline \multirow[t]{3}{*}{ Regime hídrico } & \multicolumn{7}{|c|}{ Dias após o transplante } \\
\hline & \multirow{2}{*}{$\begin{array}{c}0 \\
\text { (20 de nov.) } \\
\end{array}$} & \multirow{2}{*}{$\begin{array}{c}134 \\
\text { (3 de abr.) }\end{array}$} & \multirow{2}{*}{$\begin{array}{c}196 \\
\text { (4 de jun.) }\end{array}$} & \multirow{2}{*}{$\begin{array}{c}236 \\
\text { (14 de jul.) }\end{array}$} & \multirow{2}{*}{$\begin{array}{c}284 \\
\text { (31 de ago.) } \\
\end{array}$} & \multirow{2}{*}{$\begin{array}{c}334 \\
\text { (20 de out.) } \\
\end{array}$} & \multirow{2}{*}{$\begin{array}{c}383 \\
(9 \text { de dez. })\end{array}$} \\
\hline & & & & & & & \\
\hline & \multicolumn{7}{|c|}{$\operatorname{IAF}\left(\mathrm{cm}^{2} / \mathrm{cm}^{2}\right)$} \\
\hline Irrigado & 0,01 & $0,102 \mathrm{G}$ & $0,259 \mathrm{EF}$ & $0,291 \mathrm{E}$ & $0,445 \mathrm{D}$ & $0,772 \mathrm{~B}$ & $1,162 \mathrm{~A}$ \\
\hline \multirow[t]{2}{*}{ Não-irrigado } & 0,01 & $0,125 \mathrm{G}$ & $0,218 \mathrm{~F}$ & $0,221 \mathrm{~F}$ & $0,227 \mathrm{EF}$ & $0,290 \mathrm{E}$ & $0,663 \mathrm{C}$ \\
\hline & \multicolumn{7}{|c|}{ MS (g/planta) } \\
\hline Irrigado & 3,0 & 19,70 & $55,2 \mathrm{MN}$ & $70,9 \mathrm{LM}$ & $117,9 \mathrm{~J}$ & $195,1 \mathrm{I}$ & $270,8 \mathrm{H}$ \\
\hline \multirow[t]{2}{*}{ Não-irrigado } & 3,0 & $23,5 \mathrm{O}$ & $50,5 \mathrm{~N}$ & $58,2 \mathrm{LMN}$ & $72,5 \mathrm{~L}$ & $93,7 \mathrm{~K}$ & $183,0 \mathrm{I}$ \\
\hline & \multicolumn{5}{|c|}{ Equações ajustadas $(0 \leq \mathrm{DAT} \leq 383)$} & \multicolumn{2}{|c|}{$\mathrm{R}^{2}$} \\
\hline & \multirow{2}{*}{\multicolumn{5}{|c|}{$\operatorname{IAF}\left(\mathrm{cm}^{2} / \mathrm{cm}^{2}\right)$}} & \\
\hline Irrigado & & & & & & \multicolumn{2}{|c|}{0,995} \\
\hline \multirow[t]{2}{*}{ Não-irrigado } & \multicolumn{5}{|c|}{$\begin{array}{l}\mathrm{IAF}=4 \times 10^{-8} \mathrm{DAT}^{3}-1 \times 10^{-5} \mathrm{DAT}^{2}+0,0016 \mathrm{DAT}+0,0073 \\
\mathrm{IAF}=5 \times 10^{-8} \mathrm{DAT}^{3}-2 \times 10^{-5} \mathrm{DAT}^{2}+0,0037 \mathrm{DAT}+0,0018\end{array}$} & \multicolumn{2}{|c|}{0,938} \\
\hline & \multicolumn{5}{|c|}{ MS (g/planta) } & \\
\hline Irrigado & \multirow{2}{*}{\multicolumn{5}{|c|}{$\begin{array}{l}\mathrm{MS}=4 \times 10^{-6} \mathrm{DAT}^{3}+0,0003 \mathrm{DAT}^{2}+0,0306 \mathrm{DAT}+3,0393 \\
\mathrm{MS}=9 \times 10^{-6} \mathrm{DAT}^{3}-0,0037 \mathrm{DAT}^{2}+0,5874 \mathrm{DAT}+1,3637\end{array}$}} & \multirow{2}{*}{\multicolumn{2}{|c|}{$\begin{array}{l}0,997 \\
0,967\end{array}$}} \\
\hline Não-irrigado & & & & & & & \\
\hline
\end{tabular}

${ }^{(1)}$ Médias de cada parâmetro de crescimento seguidas de mesma letra não diferem entre si pelo teste de Duncan, a $5 \%$ de probabilidade.
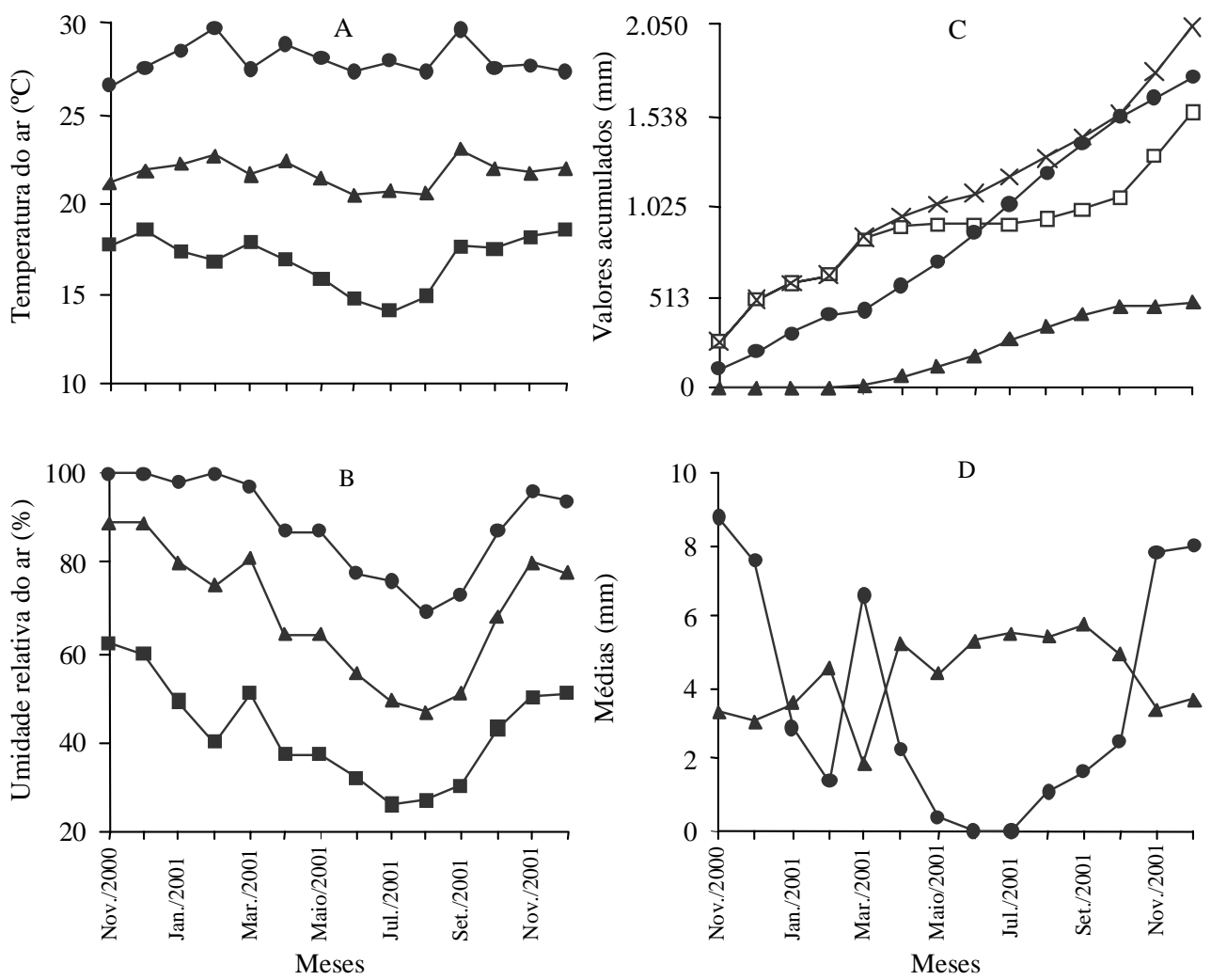

Figura 1. A: médias mensais de temperatura do ar (•: máxima; $\mathbf{\bullet}$ : mínima; $\mathbf{\Delta}$ : média); B: umidade relativa do ar (•: máxima; $\mathbf{\square}$ : mínima; $\mathbf{\Delta}$ : média); C: valores acumulados da lâmina total de água $(\times)$; da precipitação $(\square)$; da evaporação do tanque classe A $(\bullet)$; e da irrigação $(\mathbf{\Lambda})$; D: médias mensais da precipitação pluviométrica $(\bullet)$; e da evaporação do tanque classe A $(\mathbf{\Lambda})$, no período de novembro de 2000 a dezembro de 2001 no Distrito Federal. 
do, tem sido comumente associada com a época seca, que em alguns casos coincide com baixas temperaturas e dias curtos, o que dificulta a identificação do fator responsável pela variação sazonal do crescimento neste período.

As plantas não irrigadas apresentaram surto de crescimento, no período de outubro a dezembro, com a instalação do período quente e chuvoso, mostrando a habilidade da planta de recuperação do estresse termo-hídrico sofrido no inverno. Por outro lado, as que foram irrigadas durante todo o ano não apresentaram esse crescimento compensatório. Tais surtos coincidiram com o desenvolvimento das gemas em ramos secundários e em frutos. Carelli et al. (1989) citaram que cafeeiros em fase de frutificação apresentaram maior absorção de nutrientes, assim como maior taxa de fotossíntese, sugerindo que, na presença de frutos, as plantas tornam-se metabolicamente mais ativas. No entanto, crescimento compensatório em plantas jovens sem frutos foi também verificado por Tesha \& Kumar (1979). Este comportamento da taxa de crescimento sugere que outros fatores além do teor de água do solo e temperatura, provavelmente, relacionados com o balanço hormonal, influenciam o crescimento do cafeeiro no período quente e chuvoso.

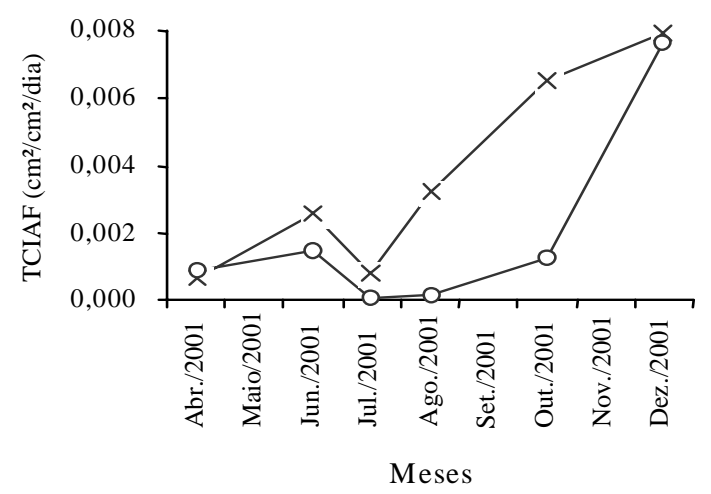

Ao final de 383 DAT, os tratamentos irrigados promoveram significativo crescimento do cafeeiro. As plantas irrigadas aumentaram, em média, $48 \%$ a massa da parte aérea, $79 \%$ o número de nós com gemas, $75 \%$ o índice de área foliar, $16 \%$ o número de ramos plagiotrópicos e $90 \%$ a massa de ramos plagiotrópicos.

A distribuição de matéria seca entre os órgãos da parte aérea do cafeeiro sem irrigação diferiu de modo acentuado da que foi apresentada pelo cafeeiro irrigado (Figura 3), principalmente no desenvolvimento de gemas, antecipando o aumento na massa de frutos. Possivelmente, com o maior desenvolvimento das gemas a partir do final de agosto nas plantas sem irrigação, o crescimento em massa do caule, folhas e ramos plagiotrópicos foi reduzido em favorecimento do enchimento dos frutos em dezembro. O mesmo não foi observado nos cafeeiros irrigados. Estes dados estão de acordo com Rena \& Maestri (2000), que indicam que os frutos demandam prioritariamente os fotoassimilados, mesmo desfavorecendo a integridade fisiológica do cafeeiro. Amaral (1991) constatou que cafeeiros apresentaram maiores taxas de crescimento quando tiveram os seus frutos removidos.

Figura 2. Taxas médias de crescimento do índice de área foliar (TCIAF) e da massa da parte aérea seca (TCMS) incluindo caule, ramos, folhas e gemas do cafeeiro Rubi MG 1192 em dois regimes hídricos ( $\times$ : irrigado; o: não-irrigado) no Distrito Federal. 

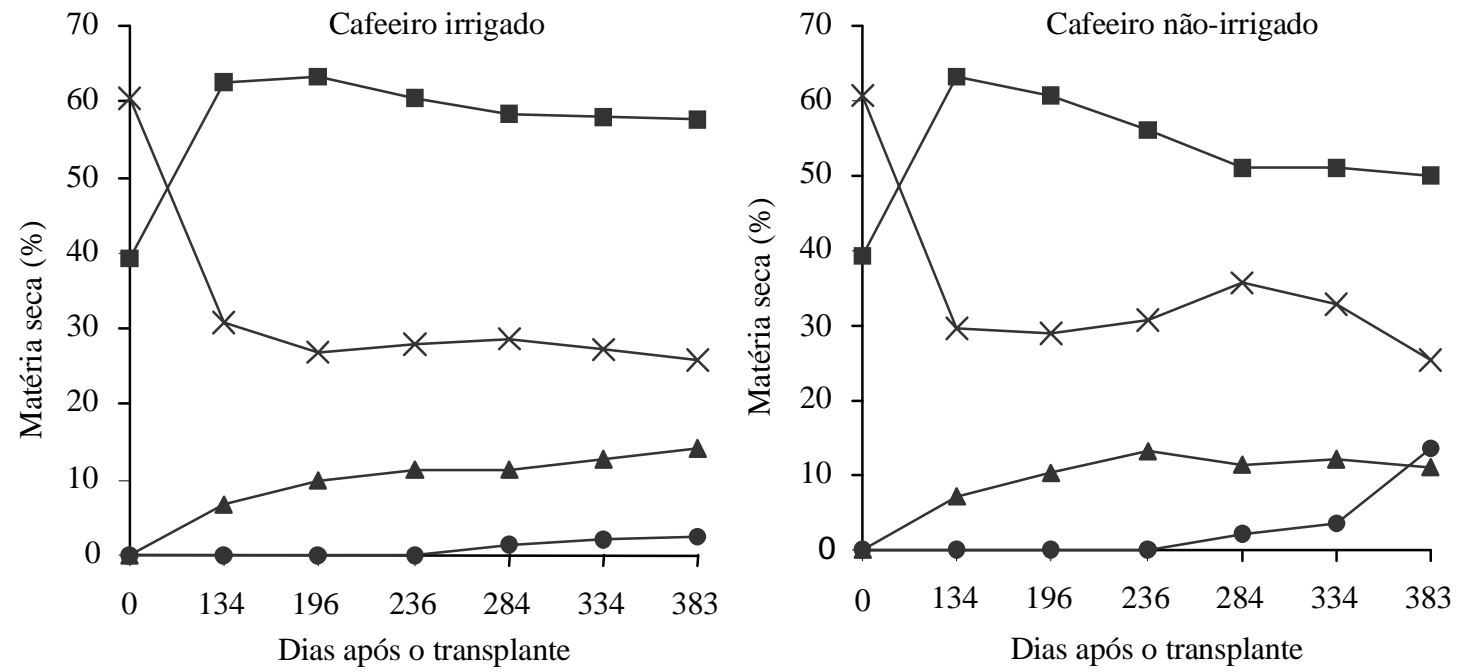

Figura 3. Distribuição de matéria seca entre caule $(\times)$, ramos $(\boldsymbol{\Delta})$, folhas $(\boldsymbol{\bullet})$ e gemas $(\bullet)$ do cafeeiro Rubi MG 1192 nos regimes hídricos irrigado e não-irrigado em razão dos dias após o transplante. As gemas incluem a massa de gemas, ramificações secundárias e frutos em estágio de enchimento.

\section{Conclusões}

1. As doses de N, Pe K não influenciam significativamente o crescimento inicial da parte aérea do cafeeiro em termos de índice de área foliar e massa de planta.

2. No cafeeiro irrigado, o crescimento inicial da parte aérea da planta, em termos do número de ramos plagiotrópicos e de número de nós com gemas por planta, é afetado significativamente pelas doses de $\mathrm{N}$ e K, mas não de fósforo.

3. A irrigação do cafeeiro favorece o crescimento da parte aérea da planta.

4. Durante o período seco e frio da região do Cerrado, os cafeeiros com e sem irrigação reduzem as taxas de crescimento.

5. O desenvolvimento das gemas em frutos altera a distribuição de matéria seca da parte aérea da planta, favorecendo os tratamentos não-irrigados em termos de massa de gemas/frutos.

\section{Referências}

AMARAL, J. A. T. do. Crescimento vegetativo estacional do cafeeiro e suas inter-relações com fontes de nitrogênio, fotoperíodo, fotossíntese e assimilação de nitrogênio. 1991. 139 f. Tese (Doutorado em Fisiologia Vegetal) - Universidade Federal de Viçosa, Viçosa, MG, 1991.

ANDROCIOLI FILHO, A. Procedimento para o adensamento de plantio e contribuição para o aumento da produtividade. In: SIMPÓSIO INTERNACIONAL SOBRE CAFÉ ADENSADO, 1994, Londrina. Anais... Londrina: Instituto Agronômico do Paraná, 1994. p. 249-275.

BARROS, R. S.; MOTA, J. W. S. e; MATTA, F. M. da; MAESTRI, M. Decline of vegetative growth in Coffea arabica $\mathrm{L}$. in relation to leaf temperature, water potential and stomatal conductance. Field Crops Research, Amsterdam, v. 54, p. 65-72, 1997.

CARELLI, M. L. C.; FAHL, J. I.; MAGALHÃES, A. C. Assimilação de nitrato durante o desenvolvimento reprodutivo de plantas de café. Revista Brasileira de Ciência do Solo, Campinas, n. 13, p. 59-64, 1989.

Pesq. agropec. bras., Brasília, v. 38, n. 8, p. 903-910, ago. 2003 
EMBRAPA. Centro Nacional de Pesquisa de Solos (Rio de Janeiro, RJ). Manual de métodos de análise de solo. 2. ed. Rio de Janeiro, 1997. 212 p.

GERVÁSIO, E. S.; LIMA, L. A. Desenvolvimento do cafeeiro (Coffea arabica L.) em função de diferentes lâminas de água aplicadas durante a fase inicial de formação da lavoura. Revista Brasileira de Engenharia Agrícola e Ambiental, Campina Grande, v. 2, n. 1, p. 68-74, 1998.

GUIMARÃES, P. T. G. Respostas do cafeeiro (Coffea arabica $L_{\text {.) }}$ à adubação mineral e orgânica em solos de baixa fertilidade do sul de Minas Gerais. 1986. $140 \mathrm{f}$. Tese (Doutorado em Solos e Nutrição de Plantas) - Escola Superior de Agricultura Luiz de Queiroz, Piracicaba, 1986.

GUIMARÃES, P. T. G.; GARCIA, A. W. R.; ALVAREZ VENEGAS, V. H.; PREZOTTI, L. C.; VIANA, A. S.; MIGUEL, A. E.; MALAVOLTA, E.; CORRÊA, J. B.; LOPES, A. S.; NOGUEIRA, F. D.; MONTEIRO, A. V. C. Cafeeiro. In: RIBEIRO, A. C.; GUIMARÃES, P. T. G.; ALVAREZ VENEGAS, V. H. (Ed.). Recomendações para o uso de corretivos e fertilizantes em Minas Gerais:

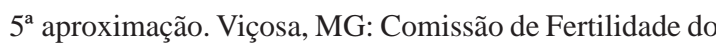
Solo do Estado de Minas Gerais, 1999. p. 289-302.

MATTA, F. M. da; AMARAL, J. A. T. do; RENA, A. B. Growth periodicity in trees of Coffea arabica $\mathrm{L}$. in relation to nitrogen supply and nitrate reductase activity. Field Crops Research, Amsterdam, v. 60, p. 223-229, 1999.

MENDES, A. N. G. Cultivares de cafeeiro e espaçamento de plantio. In: RIBEIRO, E. de S.; ABREU, E. M. Programa treina café: produção e gerenciamento do café. Lavras: Ufla/Faepe, 1999. p. 19-37.

MOTA, J. W. S. e. Fatores associados à queda sazonal do crescimento do cafeeiro arábico em Viçosa MG. 1988. 68 f. 1988. Dissertação (Mestrado em Solos e Nutrição de Plantas) - Universidade Federal de Viçosa, Viçosa, MG, 1988.
RAIJ, B. van; CANTARELLA, H.; QUAGGIO, J. A.; FURLANI, A. M. C. (Ed.). Recomendação de adubação e calagem para o Estado de São Paulo. 2. ed. Campinas: Instituto Agronômico, 1996. p. 97-101. (Boletim Técnico, 100).

RENA, A. B.; MAESTRI, M. Fisiologia do cafeeiro. In: CULTURA do cafeeiro: fatores que afetam a produtividade. Piracicaba: Potafos, 1986. p. 13-85.

RENA, A. B.; MAESTRI, R. Relações hídricas no cafeeiro. Item, Irrigação e Tecnologia, Brasília, n. 48, p. 34 41, set. 2000.

SILVA, E. A. da. Periodicidade do crescimento vegetativo em Coffea arabica $L$.: relações com a fotossíntese em condições de campo. 2000. 31 f. Dissertação (Mestrado em Fisiologia Vegetal) - Universidade Federal de Viçosa, Viçosa, MG. 2000.

SILVA, E. B. de; NOGUEIRA, F. D.; GUIMARÃES, P. T. G.; CHAGAS, S. J. de R.; COSTA, L. Fontes e doses de potássio na produção e qualidade do grão de café beneficiado. Pesquisa Agropecuária Brasileira, Brasília, v. 34, n. 3, p. 335-345, mar. 1999.

TESHA, A. J.; KUMAR, D. Effects of soil moisture, potassium and nitrogen on mineral absorption and growth of Coffea arabica L. Turrialba, San José, v. 29, n. 3, p. 213-218, jul./set. 1979.

VAAST, P.; ZASOSKI, R. J.; BLEDSOE, C. S. Effects of solution $\mathrm{pH}$, temperature, nitrate/ammonium ratios and inhibitors on ammonium and nitrate uptake by arabica coffee in short term solution culture. Journal of Plant Nutrition, Moticello, v. 21, n. 7, p. 1551-1564, 1998.

WINSTON, E. C.; LITTLEMORE, J.; SCUDAMORE S, P.; O'FARREL, P. J.; WIFFEN, D.; DOOGAN, V. J. Effect of nitrogen and potassium on growth and yield of coffee (Coffea arabica L.) in tropical Queensland. Australian Journal of Experimental Agriculture, Melbourne, v. 32, p. 217-224, 1992. 